\title{
L'uso del qual(')è nella grafia di Leonardo Sciascia
}

\section{Salvatore Claudio Sgroi}

PUBBLICATO: 9 LUGLIO 2021

$\mathrm{L}$

a grafia con apostrofo <qual'è è, com’è noto, oggetto di continue diatribe ortografiche, variamente contestata rispetto a quella, omofona, senz'apostrofo <qual è (cfr. Sgroi 2019/a pp. 39-51; Satta I968 ${ }^{\mathrm{I}}$, $1974^{2}$ pp. 29-33; 274-75; 1989 p. 263).

La [Regola-I] [R-I] alla base della grafia canonica, tradizionale, è quella del troncamento, per cui la forma piena <quale > si tronca (e non si elide) in <qual dinanzi sia a vocale che a consonante. Esattamente come nel caso di <uno/un> vs <una/un'>. Si dice infatti nel qual caso, per la qual cosa, ogni qual volta, ho una certa qual fretta, con una certa qual sorpresa, qual piuma al vento.

La [Regola-2] [R-2] alla base della grafia contestata <qual'é, qual'eras è invece quella dell'elisione, perché il troncamento di quale dinanzi a consonante è in realtà legato all'italiano dei secoli passati, e gli ess. di cui sopra sono formule pressoché fossilizzate. Nell'italiano contemporaneo, si dice infatti quale ragazzo hai invitato? e non già "qual ragazzo hai invitato?, quale film vuoi vedere? e non *qual film vuoi vedere?, ecc.

La Norma-1 della $[\mathrm{R}-\mathrm{I}]<q u a l$ é> caratterizza l'italiano standard letterario. La Norma-2 della [R-2] con apostrofo <qual'e $>$, è propria dell'italiano neo-standard, come sembra peraltro dimostrato dalla sua crescita nella rete. Stando infatti a "Google Docs", che analizza statisticamente l'uso della lingua sulla base di un algoritmo della Intelligenza Artificiale (I.A.), la grafia <qual'è (con apostrofo) risulta quella più frequente, ed è normativamente giudicata "corretta" rispetto a quella senza apostrofo, di uso minoritario (cfr. Sgroi 202I).

Sulla scorta del Coliweb, un metacorpus dell'italiano, ideato da M. Biffi, selezionato da 72 siti web, attualmente con circa 8oomila occorrenze (e un lemmario di circa 34mila lemmi), ma in espansione con previsione di "almeno 2 miliardi di parole" (Biffi-Ferrari 2020 p. 37I), la grafia con apostrofo <qual'es appare in 2,60I ess. (= pp. 27) minoritaria rispetto alla variante senz'apostrofo <qual é> presente in 19,555 ess. (= pp. I96), in un rapporto quindi di I a Io; e analogamente per quanto riguarda <qual'era $>$ in 146 ess. (= pp. 2) VS <qual era $>$ in 35I ess. (= pp. 4.), in un rapporto di I a quasi 2.

Per noi invece, entrambe le grafie, indipendentemente dalla maggioranza, sono "laicamente" corrette in quanto presenti in testi di utenti colti o mediamente colti, e anche illustri.

Per quanto riguarda la grafia sciasciana, nella "Nota al testo" (pp. I39-70) di L. Sciascia 202I 'Questo non è un racconto', P. Squillacioti 202I constata che la grafia con elisione <qual'è è un "uso scrittorio caratteristico di Sciascia almeno fino all'inizio degli anni Settanta e sistematicamente regolarizzato dalle redazioni delle case editrici" (p. I49; anche in Squillacioti $2012=2020^{2}$ p. 1947). Ovvero individua, possiamo dire, due regole ortografiche: la [Regola-I] sciasciana <qual'é e la [Regola-2] editoriale <qual $\grave{e}>$, agg. interrogativo diretto/indiretto e indefinito.

E cita al riguardo due esempi:

(i) "E qual'è la differenza" nel dattiloscritto del Consiglio d'Egitto [ig63] [Regola-I] normalizzato 
[Regola-2] in <qual é > nell'edizione einaudiana del I963 (p. 5I); e quindi in Opere I [2012=2020 $\left.{ }^{2}\right]$ p. 386 ); e

(ii) "Voi sapete qual'è la situazione politica" nel dattiloscritto del Contesto. Una parodia [197I] [RegolaI]: "anche in questo caso l'edizione einaudiana del 1971 [p. 74] normalizza [Regola-2] in 'qual è' (e quindi in Opere, vol. I [I2I2 = $\mathrm{I}_{22 \mathrm{O}^{2}}$ ] p. 67I)" (Squillacioti 202I p. I49), un uso "ancora più significativo" (Squillacioti ibid.) perché già - si sottolinea - nella lezione precedente: "Voi sapete qual'è, oggi, la situazione" poi scartata $\left(2012=202 \mathrm{O}^{2}\right.$ p. I848).

Da cui emerge che la [Regola-2] della normalizzazione editoriale è adottata anche dal filologo Squillacioti [Regola-2bis], malgrado la [Regola-I] del dattiloscritto sciasciano I963 (Squillacioti 20I2 = $2020^{(2)}$ pp. I848-49).

Un terzo esempio - presupposto dal dattiloscritto - è invece "l'apologo della chiesa qual'è oggi" nel racconto Come pensano $i$ vescovi del $\mathbf{1 9 5 8}$, stampato in "Libera stampa" e in "il Caffe politico e letterario" ried. in Racconti dispersi (Opere OA I [Sciascia 20r2]: "qual è oggi" p. I947; [2020²]: "qual'è oggi" p. I264). La [Regola-I] sciasciana, mancando il dattilo, è qui presupposta sulla scorta della stampa del 1958 , ed è quindi adottata dal filologo.

Lo stesso Squillacioti 202I riporta un quarto esempio in Per Carlo Lizzani I968 inedito, stampato nel 202I pp. I3-26: "hanno capito qual'è il vostro punto debole" (p. 23).

Squillacioti individua anche una seconda fase nell'uso scrittorio di L. Sciascia quando constata che "già all'altezza della stesura di Todo Modo [I974], Sciascia adotta la forma aferetica 'qual è' sia con referente femminile ('Qual è, la tua riflessione?'), sia maschile ('E qual è, questo filo') Opere I [ $\left.\mathrm{I}^{*} \mathrm{I} 2=\mathrm{I}_{2} 2 \mathrm{O}^{2}\right]$ pp. 932, 923; ed è poi l'unica attestata nei dattiloscritti degli anni successivi" (pp. I49-50).

La [Regola-2] <qual è> degli editori coincide quindi con quella di Sciascia [Regola-2 ter] - sulla scorta dei dattiloscritti e quindi con quella del filologo Squillacioti [Regola-2bis].

Su 25 occorrenze di $\langle q u a l(') \dot{e}>$, i cui luoghi nei 3 voll. (2012, I2I4, I2I9) ci sono stati segnalati molto generosamente (e pazientemente) da P. Squillacioti, alla fine una sola è l'occorrenza di <qual'é> secondo la [Regola-I] di L. Sciascia che compare nel vol. delle Opere I, [2020²]: "qual'è oggi" (p. I264).

Le altre 24 occorrenze - I6 ante I974 e 8 a partire da Todo Modo [I974] - sono tutte normali(zzate) [Regola-2] e adottate da Squillacioti [Regola-2bis] ${ }^{* 2 *}$.

In termini di regole, possiamo quindi affermare che la [REGOLA-I] di Sciascia è quella di scrivere, ovvero di dattilografare, almeno fino al I973, con l'apostrofo <qual'è. La [REGOLA-2/a] degli Editori è quella della sistematica normalizzazione in <qual è $>$, a cui si è adeguato - "per prudenza" [REGOLA-2/b] il filologo (anche quando i dattiloscritti indicavano: qual'e [REGOLA-I].

L'unica eccezione del filologo (col qual'è p. r264) non è un'eccezione perché il filologo ha seguito la [REGOLA-3] dei due Editori costituiti dalle due riviste 1958 (che hanno rispettato la [Regola-I] di Sciascia).

Su 24 casi in I8 (oltre i 2/3) e fino al 1973 Sciascia adotta la [Regola-I] dell'apostrofo, ma a partire dal I974 in 8 casi (meno di r/3) sembra adeguarsi alla [Regola-2] senz'apostrofo degli editori. 
Per altri problemi di ortografia come "apostrofı e accenti" Squillacioti dichiara più volte di essersi adeguato all'uso corrente, senza dar conto di tali emendazioni (2012, 2020² pp. 1973, 1942). Su altri casi di grafia cfr. Sgroi (2014 [ma: 2015], ried. 2016).

\section{Riferimenti bibliografici}

Biffi-Ferrari 2020: Marco Biff1, Angela Ferrari, Progettare e realizzare un «corpus» dellitaliano nella rete: il caso del «coliweb", in "Studi di lessicografia italiana", XXXVII, 2020, pp. 357-374.

Satta 1968', 1974²: Luciano Satta, Come si dice. Uso e abuso della lingua italiana, Firenze, Sansoni, I968. - 1989, Matita rossa e blu. Lo stato della lingua italiana nell'esame spietato ma scherzoso compiuto su 110 scrittori contemporanei, Pref. di I. Montanelli, Milano, Bompiani.

Sciascia 20ro: Leonardo Sciascia, Il fuoco nel mare. Racconti dispersi (1947-1975), a cura di Paolo Squillacioti, Milano, Adelphi; [ried. ampliata col titolo Racconti dispersi (1947-1986) in Sciascia 20I2, pp. I233-409; 2020 ${ }^{2}$, pp. I233-4IO].

- 20I2, Opere, vol. I Narrativa, Teatro, Poesia, a cura di P. Squillacioti, Milano, Adelphi, II ed. 2020.

- 2014, Opere, vol. II, Inquisizioni, Memorie, Saggi, t. I Inquisizioni e Memorie, a cura di P. Squillacioti, Milano, Adelphi.

- 2019, Opere, vol. II Inquisizioni, Memorie, Saggi, t. II Saggi letterari, storici e civili, a cura di P. Squillacioti, Milano, Adelphi.

- 2021, 'Questo non è un racconto', a cura di P. Squillacioti, Milano, Adelphi.

Sgroi 2019/a: Salvatore Claudio Sgroi, Regole e pseudo-regole: a proposito del "qual è" maschile e del "qual'e" femminile, in blog F. Raso, sabato i6 febbraio 2019.

- 2019/b, Gli Errori ovvero le Veritá nascoste, Palermo, Centro di studi filologici e linguistici siciliani.

- 2020, Grammaticografia e datazione di una pseudo-regola: <qual é > masch. vs <qual'é femm., in blog F. Raso, 6 febbraio.

- 202I, L'algoritmo della Intelligenza Artificiale (I.A), ovvero la Regola della frequenza degli usi, alla base della Norma di Google Docs, in blog F. Raso, mercoledi 23 giugno.

Squillacioti 20I0: Paolo Squillacioti, «Nota al testo», in Sciascia 20Io, pp. I8I-2Io.

- 20I2, $202 \mathrm{O}^{2}$ «Introduzione», «Cronologia», «Note ai testi», in Sciascia 20I2, pp. vii-xxix; xxxi-xlii; $1695-2016$.

- 2014, «Note ai testi», in Sciascia 20I4, pp. I24I-I43I.

- 2019, «Note ai testi», in Sciascia 2019, pp. I32I-I476.

- 202I, «Nota al testo», in Sciascia 202I, pp. I39-70.

\footnotetext{
* Sulla pseudo-regola del maschile (qual é) e del femminile (qual'é), cfr. Sgroi 2019/a, ripreso in Sgroi 2019/b pp. 46-49, e Sgroi 2020 .

**: Le indichiamo cronologicamente qui di seguito:

(i) Il giorno della civetta [I96I, Opere 20I2, 2020² vol. I p. 309]: "'E qual è, secondo lei, il canale giusto in politica?' [p. I760: dattilo?];

(ii) Consiglio d'Egitto [1963, Opere 20ı2, 2020² vol. I p. 386]: "E qual è la differenza" (ma nel dattiloscritto p. I807 <qual'e));

(iii) Filologia [1963/1964, Opere 20I2, 2020² vol. I p. 775] in Il mare colore del vino 1973 (p. I857: dattilo?)]: "qual è la strada che hanno fatto, i significati che hanno mutato";

(iv) "qual è l'origine della parola" (p. 775) [dattilo?];

(v) La Sicilia nel cinema [1963, Opere 2019 vol. II, t. II p. 479, in La corda pazza 1970 (dattilo p. 1364)]: "qual è appunto in
} 
Quasimodo";

(vi) L'onorevole [1965, Opere 2012, 2020² vol. I p. I438 (p. 1974: dattilo?)]: "e allora direi chiaro e netto qual è, dentro il partito, la mia tendenza...";

(vii) "mi ha detto qual è il motivo della sua ansia" (p. I452);

(viii) Un caso di coscienza [1966, Opere 2012, 2020² vol. I p. 796, in Il mare colore del vino 1973 (p. I88o: dattilo?)]: "ignaro qual è suo marito";

(ix) A ciascuno il suo [1966, Opere 2012, $2020^{2}$ vol. I p. $5^{23}$ (dattilo perduto p. I820, bozze irreperibili p. I825)]: "ma la tentazione,

[...], rabbiosa, infernale qual è?";

(x) "Qual è l'animale che tiene il becco sottoterra?" (p. 578);

(xi) Rapporto sulle cose siciliane [1968, Opere 2019 vol. II, t. II, p. 442, in La corda pazza 1970 (p. I363: dattilo?)]: "il destino della città qual è oggi";

(xii) Recitazione [1969, Opere 2012, 2020² vol. I pp. 1503-04 (p. 1981: dattilo?)]: "Ma sapete qual è l'episodio [...]?";

(xiii) I sei personaggi [1970, Opere 2019 vol. II, t. II p. 365, in La corda pazza $197 \mathbf{9}$ (p. I360: dattilo?)]: "Qual è l'anello che non tiene [...]?";

(xiv) Contesto. Una parodia [I97I, Opere 2012, 2020² vol. I p. 671 (ma nel dattilo <qual'és pp. I848-49; normalizzata nell'edizione einaudiana del I97I (p. 74) e nella rist. Adelphi I994, p. I849 n. I; e Squillacioti 202I p. 74)]: "Voi sapete qual'è la situazione";

(xv) I mafiosi [1972, Opere 20I2, $2020^{2}$ vol. I p. 1596 (p. 1985: dattilo?)]: "Ma qual è, o amici miei, l'autentico significato della parola mafia?";

(xvi) 'Storia della colonna infame' [1973, Opere 2019 vol. II, t. II, p. 602, in Cruciverba 1983 (p. 1384: dattilo?)]: "farne altra cosa che romanzo: qual è";

A partire da Todo modo 1974:

(xvii) Todo modo [1974, Opere 20I2, 2020² vol. I p. 923 (dattilo: p. I89I)]: "E qual è, questo filo?";

(xviii) "Qual è la tua riflessione?', domandò Sgalambri" (p. 932);

(xix) Luciano e le fedi [1974, Opere 2019 vol. II, t. II, p. 496; in Cruciverba $\mathbf{1 9}_{\mathbf{9} 3}$ (p. I371: dattilo?)]: "Ma capisce anche qual è il punto debole";

(xx) Il teatro della memoria [1981, Opere 20I4 vol. II, t. I, p. 657 (p. I336: dattilo?)]: "da grossolano qual è";

(xxi) La sentenza memorabile [1982, Opere 20I4 vol. II, t. I p. 687 (dattiloscritto "presso gli eredi" p. I342)]: "qual è certo che fosse";

(xxii) "Sciascia: responsabilità del giudice" (titolo redazionale, p. I459) ["Corriere della Sera" 7 agosto I983, Opere 2019 vol. II, t. II, in A futura memoria (se la memoria ha un futuro) $\mathbf{1 9 8 9}$ (dattiloscritto di 4 pagg. p. I459)]: "Ma qual è la buona [cattura]?" (p. I245); (xxiii) 1912+1 [1986, Opere 20I4 vol. II, t. I, p. 837 (dattilo p. I378)]: "qual è il suo nome di battesimo?";

(xxiv) Scopriamo chi ha ucciso Pinelli (titolo redazionale p. I474) ["L'Espresso" 28 agosto $\mathbf{1 9 8 8}$, in A futura memoria $\mathbf{1 9 8 9}$, in Opere 2019 vol. II, t. II (p. I475: copia carbone pp. 4)]: "So, [...], qual è la risposta" (p. г313).

\section{Cita come:}

Salvatore Claudio Sgroi, L'uso de/qual(')è nella grafia di Leonardo Sciascia , "Italiano digitale", XVIII, 2021/3 (luglio-settembre)

DOI: $10.35948 / 2532-9006 / 2021.9580$

Copyright 2021 Accademia della Crusca

Pubblicato con licenza creative commons CC BY-NC-ND 